\title{
Rumiación, preocupación y orientación negativa al problema: procesos transdiagnósticos de los trastornos de ansiedad, de la conducta alimentaria y del estado de ánimo
}

\author{
Manuel González*, Ignacio Ibáñez y Andrea Barrera \\ Universidad de La Laguna (ULL), Tenerife, España
}

Recibido, junio 21/2016

Concepto de evaluación, noviembre 10/2016

Aceptado, diciembre 12/2016
Referencia: González, M., Ibáñez, I. \& Barrera, A. (2017). Rumiación, preocupación y orientación negativa al problema: procesos transdiagnósticos de los trastornos de ansiedad, de la conducta alimentaria y del estado de ánimo. Acta Colombiana de Psicología. 20(2), 30-41. doi: http://www.dx.doi.org/10.14718/ACP.2017.20.2.3

Resumen

Este estudio presenta las relaciones existentes entre los procesos cognitivos de orientación negativa al problema, preocupación y rumiación, con la sintomatología ansiosa, depresiva y la presente en dificultades en la conducta alimentaria. Se utilizó una muestra no clínica e intencionada de 176 personas adultas de la isla de Tenerife. Los datos se analizan mediante el coeficiente de Chi-cuadrado de Pearson $\left(\chi^{2}\right)$, la $t$ de Student, el coeficiente de correlación $r$ de Pearson y regresiones jerárquicas. Se constata que la orientación negativa al problema y la preocupación son transdiagnósticos para los síntomas de agorafobia y pánico, ansiedad generalizada, fobia social y control de la comida; mientras que los reproches son comunes a la sintomatología depresiva, el estrés postraumático y la dieta, y que la fobia social también se relaciona con el proceso reflexivo. Los resultados se discuten a partir el modelo teórico de la fobia social y el estrés postraumático, y se propone la investigación futura de los reproches y la reflexión, atendiendo a la teoría de la evitación cognitiva.

Palabras clave: preocupación, rumiación, dificultades de la conducta alimentaria, transdiagnóstico.

\section{Rumination, worry and negative problem orientation: transdiagnostic processes of anxiety, eating behavior and mood disorders}

\begin{abstract}
This research presents the relationship between the cognitive processes of negative problem orientation, trait worry and rumination, with anxious and depressive symptomatology and difficulties in eating behavior. The study was conducted with a non-clinical and intentional sample of 176 adults from the island of Tenerife. Data were analyzed using the Pearson Chi-square coefficient, Student's t, Pearson's correlation coefficient, and hierarchical regressions. Results confirm that negative problem orientation and trait worry are transdiagnostic factors for panic symptoms and agoraphobia, generalized anxiety disorder, social phobia and behavioral eating, whereas brooding is a transdiagnostic factor for depression, posttraumatic stress disorder and behavioral eating. Reflection has a relationship with social phobia. Results are discussed on the basis of the theoretical model of social phobia and posttraumatic stress. Future investigation on brooding and reflection according to the theory of cognitive avoidance is proposed.

Key words: trait worry, rumination, eating disorders, transdiagnostic factors.
\end{abstract}

\footnotetext{
Departamento de Psicología Clínica, Psicobiología y Metodología. Ciencias de la Salud. Sección de Psicología. Universidad de La Laguna (ULL). Campus de Guajara, 38205. +34922 3174 65, mgonzaro@ull.es
} 


\title{
Ruminação, preocupaçáo e orientaçâo negativa ao problema: processos transdiagnósticos [RD1] dos transtornos de ansiedade, do comportamento alimentar e do bumor
}

Resumo

\begin{abstract}
Este estudo apresenta as relações existentes entre os processos cognitivos de orientação negativa ao problema, preocupação e ruminação, com a sintomatologia ansiosa, depressiva e a presente em dificuldades no comportamento alimentar. Utilizouse uma amostra não clínica e intencionada de 176 pessoas adultas da ilha de Tenerife (Espanha[RD2] ). Os dados foram analisados mediante o coeficiente de Qui-quadrado de Pearson (c2), o t de Student, o coeficiente de correlação $r$ de Pearson e regressões hierárquicas. Constata-se que a orientação negativa ao problema e a preocupação são transdiagnósticos para os sintomas de agorafobia e pânico, ansiedade generalizada, fobia social e controle da comida; enquanto a rejeição é comum à sintomatologia depressiva, ao estresse pós-traumático e ao regime. A fobia social também se relaciona com o processo reflexivo. Os resultados são discutidos a partir do [RD3] modelo teórico da fobia social e do estresse pós-traumático, e propõese a pesquisa futura das rejeições e da reflexão, atendendo à teoria da evitação cognitiva.

Palavras-chave: dificuldades do comportamento alimentar, preocupação, ruminação, transdiagnóstico.
\end{abstract}

\section{INTRODUCCIÓN}

Los pensamientos negativos repetitivos son considerados como factores de vulnerabilidad cognitiva para diversos trastornos de ansiedad, del estado de ánimo y de la conducta alimentaria (Sternheim et al., 2012). Esos pensamientos “implican una actividad cognitiva atenta, perseverante, frecuente, y relativamente incontrolable que se centran en los aspectos negativos del yo y el mundo" (Ehring \& Watkins, 2008, p.193) y se consideran, además, un factor de riesgo común o transdiagnóstico que explicaría las elevadas tasas de comorbilidad entre los diferentes trastornos emocionales (Kalmbach, Pillai \& Ciesla, 2016; McLaughlin \& Nolen-Hoeksema, 2011; Spinhoven, Drost, Van Hemert \& Penninx, 2015).

Los constructos que tienen un elemento común con los pensamientos negativos repetitivos, entre otros, son la rumiación y la preocupación (Arditte, Shaw \& Timpano, 2016; Segerstrom, Tsao, Alden \& Craske, 2000). La rumiación, en particular, predice el inicio de la depresión y desempeña un papel relevante tanto en su mantenimiento como en su recidiva (Lyubomirsky, Chancellor, Layous \& Nelson, 2014), y, según la Teoría de los Estilos de Respuestas, la rumiación se define como:

un patrón de pensamientos y conductas repetitivas que centran la atención en uno mismo, en los síntomas depresivos y en sus causas, significados y consecuencias de estos síntomas, en lugar de centrase de manera activa en una solución para resolver las circunstancias que rodean esos síntomas (NolenHoeksema \& Morrow, 1993, p.569).

La mayoría de las investigaciones consideran a la rumiación como un constructo bidimensional constituido por dos factores: la reflexión, que es definida como un proceso introspectivo que tiene como propósito participar en la solución de problemas cognitivos para aliviar el estado de ánimo bajo; y los reproches, que consisten en la rumiación negativista o la tendencia a tener reproches sobre uno mismo y las situaciones al comparar de forma pasiva la situación actual con algún estándar no alcanzado (Thanoi \& KlaininYobas, 2015; Treynor, González \& Nolen-Hoeksema, 2003). Estas dos dimensiones son tipos de rumiación diferentes, pues los reproches serían una estrategia disfuncional, mientras que la reflexión sería una funcional (Treynor et al., 2003). Particularmente, en estudios longitudinales realizados a los seis meses y al año, se observó que los reproches se asociaron a niveles más elevados de depresión tanto al inicio como a largo plazo, mientras que la reflexión se asoció a un mayor grado de depresión inicial, pero a un grado menor a largo plazo (Treynor et al., 2003).

Si bien la rumiación se considera un factor de vulnerabilidad para la depresión, las investigaciones sugieren que se relaciona también con los trastornos de ansiedad y de la conducta alimentaria (Holm-Denoma \& Hankin, 2011; Nolen-Hoeksema, Wisco \& Lyubomirsky, 2008). Así, las investigaciones transversales corroboran las relaciones más elevadas de los reproches con depresión, agorafobia y pánico, preocupación y afecto negativo, mientras que con respecto a la reflexión se encuentran relaciones más bajas (Hasegawa, Hattori, Nishimura \& Tanno, 2015; McEvoy \& Brans, 2013; Watkins, 2009).

Adicionalmente a la rumiación, otro de los pensamientos repetitivos negativos es la denominada preocupación, que se define como:

una cadena de pensamientos o actividad lingüística verbal e imágenes (aunque más bien las primeras) cargados de afecto negativo y relativamente incontrolables. El proceso de preocupación representa un intento de solución mental de problemas sobre un 
tema cuyo resultado es incierto, aunque conlleva la posibilidad de una a más consecuencias negativas. Por lo tanto, la preocupación está muy relacionada con el proceso del miedo (Borkovec, Robinson, Pruzinsky \& DePree, 1983, p.10).

Al igual que la rumiación, la preocupación es una característica central del trastorno de ansiedad generalizada, aunque también es frecuente en otros trastornos de ansiedad y del estado de ánimo (Boelen, Reijntjes \& Smid, 2016; González, Ibáñez \& Cubas, 2006; McEvoy, Watson, Watkins \& Nathan, 2013).

La rumiación y la preocupación, aunque son constructos distintos, tienen ciertas similitudes, y los coeficientes de correlación entre ambos oscilan entre .37 y .55 (D'Hudson, Lauren \& Saling, 2010; Hasegawa et al., 2015; McEvoy \& Brans, 2013). Adicionalmente, ambos comparten el hecho de ser formas de pensamientos perseverantes con dificultades para dejar de centrar la atención en lo negativo; y tienen como consecuencias déficits en el desempeño personal, las dificultades de concentración y la atención (Watkins, Moulds \& Mackintosh, 2005). Pero a pesar de estas similitudes, constituyen dos procesos distintos (Nolen-Hoeksema et al., 2008); por ejemplo, una de las diferencias se refiere a la orientación temporal, pues las preocupaciones tienden a centrarse en eventos futuros y en posibles amenazas, mientras que la rumiación suele referirse a sucesos del pasado y el fracaso (McLaughlin, Borkovec \& Sibrava, 2007). Asimismo, se observan diferencias topográficas en sus contenidos, pues la preocupación se relaciona más con la resolución de problemas, mientras que la rumiación está más centrada en temas de pérdida, significado y autoestima.

Adicionalmente, la preocupación se relaciona con la evitación del contenido amenazante, mientras que la rumiación implica una relevancia del contenido para la persona, con una necesidad de comprender lo sucedido. El afecto con el que se asocian ambos constructos también es distinto, pues la preocupación se caracteriza por la ansiedad, y la rumiación, por la depresión (McLaughlin et al., 2007). Aunque en la investigación de la ansiedad y la depresión se hayan investigado de manera separada, la preocupación y la rumiación, sin embargo, han demostrado relaciones equivalentes con ambos trastornos (McEvoy et al., 2013).

Por otro lado, se ha señalado que la rumiación y la preocupación son factores de riesgo y de mantenimiento para la depresión y la ansiedad, de manera que las personas continúan rumiando o preocupándose a pesar de sus consecuencias negativas (Dickson, Ciesla \& Reilly, 2012) y no buscan soluciones activas para resolver los problemas que dan lugar a dichos procesos, sino que se quedan adheridas a los problemas y sentimientos implicados y no hacen nada al respecto; de este modo, tanto la preocupación como la rumiación interfieren en la resolución de problemas, lo que a su vez obstaculiza la aplicación de estrategias exitosas para superar el estado de ánimo negativo.

Un proceso cognitivo relacionado tanto con la preocupación como con la rumiación es la orientación negativa al problema (Hasegawa et al., 2015), que se define como "un sistema de creencias que reflejan la percepción de un problema como una amenaza para el bienestar, experimentando dudas sobre la capacidad de resolución de problemas, y la tendencia a ser pesimistas sobre su resultado" (Robichaud \& Dugas, 2005, p.391). En una muestra de adultos, dicha orientación negativa al problema resultó ser un predictor de la depresión(González, Peñate, Bethencourt \& Rovella, 2004) y en otros trabajos se encontraron relaciones más elevadas con la depresión, la preocupación, la agorafobia y el pánico (Fergus, Valentine, Wu \& McGrath et al., 2015; Hasegawa et al., 2015); por tanto, resultaría ser un proceso transdiagnóstico para algunos de los trastornos emocionales (Fergus et al., 2015).

Si se tiene en cuenta el género, las mujeres tienen dos veces más probabilidades que los hombres de presentar un trastorno depresivo (Kessler, 2006); diferencias que son claras en la adolescencia y la adultez, aunque en personas mayores no se han encontrado diferencias en depresión por género (Serra \& Irizaray-Robles, 2015). Por otra parte, la mayor prevalencia de la depresión en mujeres se ha explicado teniendo en cuenta varias teorías, entre las que se encuentra la teoría de los estilos de respuestas, que sugiere que las mujeres tienen un mayor estilo rumiativo que las hace más vulnerables a la depresión (Lyubomirsky et al., 2014). En este sentido, los reproches son más elevados en las mujeres (Treynor et al., 2003), quienes también tienen puntajes más altos en depresión, orientación negativa al problema y reproches (Hasegawa et al., 2015).

A pesar de lo anterior, en otra investigación no se encontraron diferencias por género en rumiación, en reproches y en reflexión (Watkins, 2009); y en un estudio de metaanálisis los resultados indicaron un tamaño de efecto pequeño (pero significativo) entre los estudios en relación al género para la rumiación $(\mathrm{d}=.24)$, los reproches $(\mathrm{d}=.19)$ y la reflexión ( $d=.17)$ (Johnson \& Whisman, 2013). Estos resultados parecen ser limitados en su magnitud, lo que indica que la teoría de los estilos de respuesta no se encuentra apoyada por dicho metaanálisis. Adicionalmente, cabe resaltar que en otros estudios se han reportado diferencias de género en las manifestaciones de los síntomas clínicos de la depresión mayor (Londoño, Peñate \& González, 2016).

Por otro lado, las dificultades de la conducta alimentaria y los síntomas de ansiedad presentan una elevada comorbilidad (Sternheim et al., 2012), pues dos tercios de las personas con dificultades de la alimentación tienen algún tipo de sintomatología ansiosa (Jordan et al., 2008). En un 
estudio (Rawal, Park \& Williams, 2010), se comparó una muestra subclínica de personas con trastornos alimentarios con otra de controles sanos, y se constató que los primeros tienen mayor nivel de rumiación depresiva, evitación experiencial y creencias acerca de los beneficios de la rumiación, lo que sugiere que la rumiación puede estar asociada con la evitación experiencial. También, en otra investigación se encontró que los reproches se relacionaron de manera específica con los trastornos de la conducta alimentaria y la depresión (Danzilo, Rieger, Palermo, Byrne \& Bell, 2016).

Dada la elevada comorbilidad entre ansiedad y depresión, y entre la primera y los trastornos de la conducta alimentaria, la dilucidación sobre cómo los procesos cognitivos son compartidos o específicos puede ofrecer una comprensión de los trastornos psicopatológicas evaluados. En este sentido, en la presente investigación se proponen dos objetivos: por un lado, determinar los procesos transdiagnósticos relacionados con los trastornos psicopatológicos evaluados mediante cuestionarios en una muestra intencionada de personas adultas de la comunidad; y, por otro, conocer la existencia de diferencias de género en las variables de procesos cognitivos y psicopatológicos.

\section{MÉTODO}

\section{Muestra}

La muestra que constituyó esta investigación fue de 176 personas adultas de la comunidad, $67 \%$ mujeres y $33 \%$ hombres. La media de edad fue de 31.2 años (DT = 13.3), con una moda de 22 y un rango que oscila entre los 18 y 72 años. Respecto al nivel de estudios, el $10.3 \%$ tenía estudios primarios, $51.9 \%$ estudios medios, $14.1 \%$ estudios de diplomatura y $23.7 \%$ estudios de licenciatura. En relación al estado civil, $63.9 \%$ eran solteros, $30.1 \%$ casados, $4.2 \%$ separado/divorciado y $1.8 \%$ viudos. Y en cuanto a su residencia, $74.4 \%$ vivían en zonas urbanas y $26.6 \%$ en zonas rurales.

\section{Instrumentos}

Escala de Respuestas Rumiativas (Nolen-Hoeksema \& Morrow, 1991). Es una escala de 22 ítems de cinco puntos que van desde "totalmente en desacuerdo" a "totalmente de acuerdo". En este estudio se emplearon los 10 ítems que evalúan los factores de reproches $(\alpha=.80)$ y reflexión $(\alpha=.74)$, pues los 12 ítems de la rumiación depresiva contienen elementos comunes con el Inventario de Depresión de Beck, de manera que podrían solaparse con los síntomas depresivos y sobrestimar de forma artefactual su capacidad predictiva (Segerstrom et al., 2000).

Inventario de Preocupación del Estado de Pensilvania (Meyer, Miller, Metzger \& Borkovec, 1990). Es un inventario de 16 ítems que evalúa la preocupación rasgo; cuyas respuestas se evalúan mediante escalas de intervalo, que van de 1 (nada) a 5 (mucho). De los 16 items del cuestionario, cinco estan redactados negativamente y pueden considerarse como un artefacto estadístico, en vez de un constructo signifcativo; esto afectaría negativamente a las propiedades psicométricas del inventario, por lo que algunos autores no consideran incluir dichos ítems (Rodebaugh, Woods \& Heimberg, 2007). En conclusión, en este trabajo solo se incluyeron los 11 ítems en positivo, cuya consistencia interna oscila entre .84 y .91 .

\section{Cuestionario de Orientación Negativa al Problema.}

Consta de 12 ítems en una escala Likert de 5 puntos que van de 1 (en absoluto característico de mí) a 5 (extremadamente característico de mí). Este cuestionario evalúa la forma en que la persona reacciona o piensa cuando se encuentra con problemas, y cuenta con una consistencia interna de .95 y fiabilidad test-retest de .80 (Robichaud \& Dugas, 2005).

Inventario de Ansiedad de Beck (Beck, Epstein, Brown $\&$ Steer, 1988). Es un inventario de 21 ítems que evalúa el nivel de gravedad de la agorafobia y el pánico. La persona tiene que elegir, entre cuatro alternativas $(0=$ ninguno y $3=$ grave), el grado de molestia que le ha provocado cada síntoma durante los últimos siete días. Su fiabilidad test-retest es de .75 y la consistencia interna, de .92 . En la calificación, los criterios de gravedad son los siguientes: 0-7 = normal; $8-15=$ leve; $16-25=$ moderada y $26-63=$ severa (Beck, Epstein, Brown \& Steer, 1988).

Inventario de Depresión de Beck (Beck, Steer \& Brown, 1996). Consta de 21 ítems para los que la persona tiene que elegir, entre cuatro alternativas, la frase que mejor describe su estado de ánimo; cada ítem se valora de 0 a 3 ; y su consistencia interna es de.89. Los criterios de gravedad en la calificación son: 0-13 = normal; 14-19= leve; 20-28 = moderada; y 2963 = grave (Beck, Steer \& Brown, 1996).

Evaluación del Trastorno de Ansiedad Generalizada $(G A D-7)$. Es un instrumento que evalúa el trastorno de ansiedad generalizada mediante siete ítems. En este, se le pregunta a los participantes por la presencia de una serie de síntomas durante las dos últimas semanas, y se responde según una escala tipo Likert de cuatro puntos que van de 0 
a 3 (nunca, varios días, la mitad de los días y casi a diario). $\mathrm{Su}$ consistencia interna es de $.92 \mathrm{y}$ su fiabilidad tests-retest de .83. Las puntuaciones finales van de $0-9=$ ansiedad leve, 10-14 = ansiedad moderada y 15-21 = ansiedad severa (Spitzer, Kroenke, Williams \& Löwe, 2006).

Cuestionario de Fobia y Pánico de Albania (Brown, White \& Barlow, 2005). Es un cuestionario de 27 ítems que evalúa la agorafobia, la fobia social y los síntomas interoceptivos. Los participantes responden en una escala de 0 (ningún miedo) y 8 (miedo extremo). Para este trabajo solo se utilizó el factor de fobia social, que contiene 10 ítems, y que cuentan con una consistencia interna de .89. El rango final va de $0-80$, y en una muestra clínica, la media es igual a $21.49(\mathrm{DT}=16.13)$.

Cuestionario de Cribado de Diagnóstico Psiquiátrico (Zimmerman \& Mattia, 2001). En este trabajo se utilizó la versión española de Pérez, García, De Vicente y Oliveras (2010), aunque solo se tomaron los ítems que evalúan el trastorno por estrés postraumático $(\alpha=.92)$, donde, con un punto de corte $\geq 10$, se sospecha de un posible caso.

Test de Actitudes hacia la Alimentación (EAT-26) (Garner, Olmsted, Bohr \& Garfinkel, 1982). Este test evalúa un amplio rango de actitudes y comportamientos relacionados con los trastornos alimentarios. Consta de 26 ítems que corresponden a tres escalas: dieta $(\alpha=.88)$; bulimia $(\alpha=.84)$ y control oral $(\alpha=.70)$. Las respuestas son tipo Likert de seis puntos; y su consistencia interna total oscila entre .70 y .88 (Doninger, Enders \& Burnett, 2005). Una puntuación mayor a 20 puede señalar algún problema con la comida, y la puntuación media en personas sanas para dieta es de $4.08(\mathrm{DT}=.3)$; para bulimia, de $.2(\mathrm{DT}=.0)$; y para control, de $2.8(\mathrm{DT}=.4)$ (Garner, Olmsted, Bohr \& Garfinkel, 1982).

\section{Procedimiento}

Como parte del programa de prácticas voluntarias de la asignatura de Evaluación Psicológica de tercer año de Psicología se formaron a 20 alumnos para la administración de las distintas pruebas con el fin de que desempeñaran el papel de evaluadores. Se les solicitó que seleccionaran de su entorno cercano a un grupo de entre ocho a diez adultos mediante el efecto de bola de nieve, estrategia que suele emplearse para recoger información de poblaciones difíciles de muestrear (Thomson, 2002); estos adultos participantes fueron de la comunidad, es decir, de fuera de los contextos exclusivamente universitarios, lo que da lugar a una mayor validez ecológica del estudio. Una vez contactados los participantes, los evaluadores les informaban del carácter experimental del trabajo y se les pedía su participación voluntaria junto con la firma del consentimiento informado.

\section{Análisis estadísticos}

Para comprobar la normalidad de los datos se emplearon los procedimientos de Shapiro-Wilk y Kolgomorov-Smirnov y la inspección visual para la muestra total y según el género. En ambos casos se confirmó la normalidad. Para conocer la existencia de diferencias de género en las variables sociodemográficas se realizó el coeficiente de Chi-cuadrado de Pearson $\left(\chi^{2}\right)$, ya que se trataba de variables categóricas; mientras que para el análisis de la edad se empleó la $t$ de Student, dado que son dos poblaciones independientes.

Debido al carácter continuo de las variables y su normalidad, se realizan los coeficientes de correlación ( $r$ de Pearson), primero entre las variables predictoras entre sí, y luego de estas últimas con los síntomas de ansiedad, depresión y dificultades de la conducta alimentaria, previa comprobación del supuesto de linealidad (contrastado mediante los gráficos de dispersión). Para cada par de variables que se sometieron a análisis correlacional, y se calcularon los coeficientes de consistencia interna ( $\alpha$ de Cronbach) en cada uno de los factores.

También, se realizaron las puntuaciones $\mathrm{Z}$ de Fisher (1925) de las correlaciones independientes para comparar la magnitud de los coeficientes de correlación entre los reproches y la reflexión con las variables psicopatológicas. Por otro lado, se realizó una diferencia de medias ( $t$ de Student) para hombres y mujeres entre variables psicopatológicas y de procesos, teniendo en cuenta, o no, la homogeneidad de varianzas mediante la prueba de Levene.

Finalmente, se optó por una regresión jerárquica para aportar información sobre el peso explicativo de cada variable independiente (reproches, reflexión, preocupación y orientación negativa al problema) sobre las dependientes (dificultades de la conducta alimentaria, ansiedad y depresión). Previo a los análisis de regresión, se realizó un estudio sobre la colinealidad de las variables independientes mediante la tolerancia, el factor de inflación y los índices de condición. En este caso, la rumiación fue el factor que pudo descomponerse en dos subfactores: la reflexión y los reproches. El resto de variables fueron globales en sí mismas y disponían de una inflación de la varianza adecuada; por lo tanto, las regresiones se efectuaron en tres bloques: en el primero se introdujeron las preocupaciones; en el segundo, la orientación negativa al problema; y en el tercero, la reflexión y los reproches en conjunto. 


\section{RESULTADOS}

Con respecto a las variables sociodemográficas, no se encontraron diferencias estadísticamente significativas $\left(\chi^{2}\right)$ por género en cuanto a nivel de estudios, estatus laboral, estado civil y lugar de residencia, ni tampoco en la edad.

\section{Análisis correlacionales}

En la Tabla 1 se observan los coeficientes de correlación entre las variables de procesos, donde la reflexión y los reproches comparten un $33 \%$ de la varianza. La orientación negativa al problema muestra los coeficientes más elevados al cruzarse con la preocupación y los reproches; los coeficientes más bajos los tuvo con la reflexión. En general, los coeficientes de consistencia interna de los cuatro constructos son altamente satisfactorios.

En la Tabla 2 se observan los coeficientes de correlación entre los procesos y las variables psicopatológicas. Así, la orientación negativa al problema, la preocupación rasgo y los reproches muestran los coeficientes más elevados con el trastorno de ansiedad generalizada, la depresión, la agorafobia y pánico, la fobia social y el trastorno de estrés postraumático, y se encuentran los coeficientes más bajos, aunque estadísticamente significativos $(\mathrm{p} \leq .05)$, con la dieta y el control. En cuanto a la reflexión, se observan correlaciones por encima de .30 con la fobia social, la depresión y el trastorno de ansiedad generalizada; los coeficientes de correlación más bajos se presentan con la agorafobia y pánico; y se encuentra una ausencia de significación estadística con las dificultades con la comida.

El test $Z$ de Fisher, que se empleó para conocer si existen diferencias en los coeficientes de correlación entre reproches y reflexión con las variables de síntomas evaluadas, muestra diferencias estadísticamente significativas en los síntomas de ansiedad y depresión, pero no en la fobia social ni en las dificultades de la conducta alimentaria; esto da cuenta de la independencia de los dos factores de la rumiación. Los coeficientes de consistencia interna de los constructos evaluados son excelentes, excepto para control, que son más bajos.

También, se observan las puntuaciones medias y rangos de cada una de las variables psicopatológicas, en las cuales se encuentra que todos los casos esas puntuaciones medias se distribuyen según una asimetría positiva dado que, en

Tabla 1

Coeficientes de correlación de orden cero entre las variables de procesos y sus coeficientes de consistencia interna $(\alpha$ de Cronbach) $N=176$

\begin{tabular}{ccccc}
\hline & REP & REF & PSWQ & $\alpha$ \\
\hline ONP & $.64^{* * *}$ & $.31^{* * *}$ & $.67^{* * *}$ & .94 \\
REP & & $.58^{* * *}$ & $.68^{* * *}$ & .83 \\
REF & & $.38^{* * *}$ & .75 \\
PSWQ & & & .90 \\
\hline
\end{tabular}

Nota $. \mathrm{ONP}=$ Orientación negativa al problema $; \mathrm{REP}=$ Reproches, $\mathrm{REF}=$ Reflexión $; \mathrm{PSWQ}=$ Rasgo de preocupación $; \alpha=\mathrm{Alfa}$ de Cronbach; $* * *=\mathrm{p} \leq .001$.

Tabla 2

Coeficientes de correlación entre los procesos cognitivos y variables psicopatológicas; consistencia interna ( $\alpha$ de Cronbach) de cada uno de los factores, media y desviación típica

\begin{tabular}{ccccccccc}
\hline & FOB & DIE & CON & BUL & TAG & BDI-II & BAI & TEP \\
\hline ONP & $.45^{* * *}$ & $.17^{*}$ & $.19^{*}$ & .09 & $.60^{* * *}$ & $.57^{* * *}$ & $.57^{* * *}$ & $.42^{* * *}$ \\
PSWQ & $.44^{* * *}$ & $.21^{* *}$ & $.15^{*}$ & .11 & $.67^{* * *}$ & $.52^{* * *}$ & $.58^{* * *}$ & $.39^{* * * *}$ \\
REP & $.40^{* * *}$ & $.29^{* * *}$ & $.19^{*}$ & $.15^{*}$ & $.59^{* * *}$ & $.64 * * *$ & $.50^{* * *}$ & $.54^{* * * *}$ \\
REF & $.35^{* * *}$ & .14 & .11 & .12 & $.32^{* * *}$ & $.33^{* * *}$ & $.27^{* * *}$ & $.31^{* * * *}$ \\
Z & .54 & 1.09 & .76 & .47 & $3.23 * * *$ & $3.88^{* * *}$ & $2.5^{* *}$ & $2.65^{* *}$ \\
$\alpha$ & $.85(10)$ & $.87(13)$ & $.66(7)$ & $.75(6)$ & $.90(8)$ & $.92(21)$ & $.93(21)$ & $.90(13)$ \\
Media & 11.1 & 3.6 & 1.7 & 3.4 & 5.7 & 9.5 & 8.3 & 2.2 \\
(DT) & $(10.3)$ & $(4.5)$ & $(2.4)$ & $(1.1)$ & $(4.9)$ & $(8.7)$ & $(9.5)$ & $(3.3)$ \\
Rango M & $0-40$ & $0-27$ & $0-12$ & $2-13$ & $0-21$ & $0-42$ & $0-56$ & $0-13$ \\
Rango C & $0-80$ & $0-39$ & $0-18$ & $0-18$ & $0-21$ & $0-63$ & $0-63$ & $0-13$ \\
\hline
\end{tabular}

Nota . ONP = Orientación negativa al problema; REP $=$ Reproches, REF $=$ Reflexión; PSWQ $=$ Rasgo de preocupación; FOB $=$ Fobia Social, $\mathrm{DIE}=$ Dieta; $\mathrm{TAG}=$ Trastorno de ansiedad generalizada; $\mathrm{CON}=$ Control; $\mathrm{BUL}=$ Bulimia; $\mathrm{BDI}-\mathrm{II}=$ depresión: $\mathrm{BAI}=$ Agorafobia y pánico; TEP $=$ Trastorno de estrés postraumático; $\alpha=$ alfa de Cronbach; $\mathrm{M}=$ Esta muestra; $\mathrm{C}=$ Cuestionario; entre paréntesis $=$ número de ítems; $\mathrm{Z}=$ test $\mathrm{Z}$ de Fishers; ${ }^{*} \mathrm{p} \leq .05 ; * * \mathrm{p} \leq .01 ; * * * \mathrm{p} \leq .001$. 
el caso del BDI-II las puntuaciones (teniendo en cuenta \pm 1 DT) van desde 1.1 hasta 18 , es decir, un criterio entre normal y leve en depresión; en el TEP la puntuación está muy por debajo del punto de corte establecido de $\geq 10$; en el TAG, el rango está en una puntuación leve; en el BAI la puntuación media se sitúa entre normal y moderado; en el EAT-26 es de 8.74 (DT = 6.89), con un rango que va de 1.85 a 15.63 , muy por debajo del punto de corte de 20; y para dieta y control, se sitúa en la media de las personas sanas, por el contrario, en bulimia es más elevada.

\section{Diferencias de medias según el género}

En la Tabla 3 se presentan las diferencias de medias estadísticamente significativas entre hombres y mujeres, donde las mujeres obtuvieron puntuaciones medias más elevadas en dieta, el trastorno de ansiedad generalizada, el trastorno de estrés postraumático, la agorafobia y pánico, y en preocupación, aunque las $\eta^{2}$ son muy pequeñas. Sin embargo, no se dan esas diferencias en fobia social, depresión, reflexión, reproches, ni en orientación negativa al problema.

Tabla 3

Diferencia de medias y desviaciones típicas y eta-cuadrado para el tamaño del efecto por género en las variables evaluadas $(N=176)$

\begin{tabular}{|c|c|c|c|c|c|c|}
\hline & \multicolumn{6}{|c|}{ Hombres $(\mathrm{N}=58)$ Mujeres $(\mathrm{N}=118)$} \\
\hline & $\mathrm{M}$ & $\mathrm{Dt}$ & $\mathrm{M}$ & $\mathrm{Dt}$ & $\mathrm{t}$ & $\prod^{2}$ \\
\hline TAG & 4.94 & 4.73 & 6.86 & 5.49 & $-2.22 *$ & .17 \\
\hline TEP & 1.31 & 2.54 & 2.66 & 3.54 & $-2.59 *$ & .19 \\
\hline BAI & 6.10 & 8.50 & 9.44 & 9.91 & $-2.20^{*}$ & .16 \\
\hline DIE & 9.02 & 7.54 & 16.27 & 10.65 & $-3.87 * * *$ & .28 \\
\hline PSWQ & 18.98 & 7.21 & 21.82 & 7.74 & $-2.30 *$ & .17 \\
\hline
\end{tabular}

Nota. PSWQ $=$ Rasgo de preocupación; DIE $=$ Dieta; TAG $=$ Trastorno de ansiedad generalizada; $\mathrm{BAI}=$ Agorafobia y pánico; TEP $=$ Trastorno de estrés postraumático; $*=\mathrm{p} \leq .05$; $* * *=\mathrm{p} \leq .001$.

\section{Análisis de regresión jerárquica}

En la Tabla 4 se observa que para la fobia social únicamente el tercer bloque no mejora significativamente la varianza explicada, aunque sí lo hace en los bloques restantes, donde alcanza un $30 \%$. Dentro de los bloques que aparecen, la preocupación, la orientación negativa al problema y la reflexión son significativas, pero no se encuentra lo mismo con respecto a los reproches.

Con respecto al TAG, con una varianza ajustada del $45 \%$, los tres bloques son relevantes (en menor medida el tercero), y todas las variables son significativas, salvo en la reflexión. En cuanto a la dieta, el único bloque que no aporta una mejoría significativa en la varianza explicada es el de la orientación negativa, que finalmente se sitúa en el $8 \%$. Los reproches son los únicos relevantes en el modelo final, pues la preocupación solo es relevante si no se tienen en cuenta otras variables. En cuanto al control, solo el primer bloque (definido exclusivamente por el PSWQ) mejora significativamente la varianza, puesto que lo sitúa finalmente en un $3 \%$; sin embargo, en el modelo final, ninguna variable es relevante; aunque en el primer bloque (el que sí resultó significativo) puede concluirse la relevancia de las preocupaciones. Por otro lado, en cuanto a la bulimia, ningún bloque mejora la varianza explicada y no se encuentra ninguna variable significativa en ninguno de los pasos.

En lo que a la sintomatología depresiva refiere, todos los bloques obtienen un incremento significativo en $R^{2}$ que se sitúa finalmente en $42 \%$. Sin embargo, en el modelo final únicamente son relevantes los reproches y la orientación negativa al problema. En cuanto a la agorafobia y pánico, tanto los bloques de la preocupación como el de la orientación negativa son relevantes, pues presentan una varianza ajustada del $37 \%$; aunque este no es el caso de los reproches ni la reflexión. Finalmente, en el estrés postraumático, cada bloque aporta un incremento significativo en $R^{2}$, que, una vez ajustado, se traduce en un $27 \%$ de la varianza, aunque solo los reproches son significativos en el modelo final.

Teniendo en cuanta estos resultados, a continuación se especifican las variables que podrían identificarse como transdiagnósticas y los síntomas en los que se reflejan.

Con respecto a la preocupación, esta es transdiagnóstica para todos los casos, aunque varía en un mayor o menor peso según se tengan o no presentes otras variables; esto excepto en la bulimia; y, retomando la relevancia, cuando se contempla en conjunto con otros predictores, pierde peso para las dificultades de la alimentación y el trastorno de estrés postraumático (al igual que para el resto de los trastornos con sintomatología ansiosa). Por su parte, la orientación negativa al problema no es transdiagnóstica para las dificultades de la alimentación; y cuando se contempla conjuntamente con otras variables, solo deja de ser relevante para el estrés postraumático. Mientras que, con respecto a los reproches, parece que estos son transdiagnósticos a la ansiedad generalizada, la dieta, los síntomas depresivos y el estrés postraumático.

Con respecto a la preocupación, esta no es relevante en el tercer bloque para ninguno de los casos, salvo para la ansiedad generalizada. También, la orientación negativa al problema deja de ser transdiagnóstica en el tercer bloque para dieta y estrés postraumático; aunque, por su parte, la reflexión no parece transdiagnóstica al ser solo relevante 
Tabla 4

Análisis de regresión jerárquica

\begin{tabular}{|c|c|c|c|c|c|c|}
\hline Variables & Bloque & Predictor & $\Delta R^{2}$ & Cambio en F & $\beta$ & $t$ \\
\hline \multirow{6}{*}{ FOB } & 1 & PSWQ & .25 & $54.54_{1106} * * *$ & .50 & $7.38^{* * *}$ \\
\hline & 2 & PSWQ & .28 & $9.17_{(1,161)}^{(1,162)} * * *$ & .33 & $3.84 * * *$ \\
\hline & 3 & PSWQ & .30 & 2.79 & .28 & $3.02 * * *$ \\
\hline & & ONP & & & .24 & $2.62 * *$ \\
\hline & & REP & & & -.01 & -.11 \\
\hline & & REF & & & .17 & $2.13^{*}$ \\
\hline \multirow{7}{*}{ TAG } & 1 & PSWQ & .38 & $102.64 * * *$ & .61 & $10.13^{* * *}$ \\
\hline & 2 & PSWQ & .44 & $18.70_{(1,166)}^{(1,167)} * * *$ & .41 & $5.55 * * *$ \\
\hline & & ONP & & & .32 & $4.32 * * *$ \\
\hline & 3 & PSWQ & .45 & 3.11 * * & .33 & $4.10^{* * *}$ \\
\hline & & ONP & & & .24 & $2.97 * * *$ \\
\hline & & REP & & & .20 & $2.20 *$ \\
\hline & & REF & & & -.00 & -.030 \\
\hline \multirow{7}{*}{ DIE } & 1 & PSWQ & .06 & $8.62_{(1,131)} * * *$ & .25 & $2.93 * * *$ \\
\hline & 2 & PSWQ & .05 & $.17_{(1,130)}^{1131)}$ & .21 & 1.87 \\
\hline & & ONP & & & .05 & .41 \\
\hline & 3 & PSWQ & .08 & $3.04_{(2,128)} *$ & .11 & .92 \\
\hline & & ONP & & & -.09 & -.76 \\
\hline & & REP & & & .33 & $2.33^{*}$ \\
\hline & & REF & & & -.04 & -.41 \\
\hline \multirow{7}{*}{$\mathrm{CON}$} & 1 & PSWQ & .03 & $6.26_{(1,163)} * *$ & .19 & $2.50 *$ \\
\hline & 2 & PSWQ & .04 & $2.05_{(1,162)}^{(1,163)}$ & .10 & 1.12 \\
\hline & & ONP & & & .13 & 1.43 \\
\hline & 3 & PSWQ & .03 & .58 & .06 & .59 \\
\hline & & ONP & & & .09 & .84 \\
\hline & & REP & & & .11 & .94 \\
\hline & & REF & & & -.00 & -.00 \\
\hline \multirow{6}{*}{ BUL } & 1 & PSWQ & .01 & 2.97 & .13 & 1.72 \\
\hline & 2 & PSWQ & .01 & $.06_{(2,164)}^{(1,165)}$ & .11 & 1.20 \\
\hline & & ONP & & & .02 & .24 \\
\hline & 3 & PSWQ & .01 & $.93_{(2,163)}$ & .06 & .56 \\
\hline & & $\begin{array}{l}\text { ONP } \\
\text { REP }\end{array}$ & & & $\begin{array}{l}-.02 \\
.12\end{array}$ & $\begin{array}{r}-.26 \\
99\end{array}$ \\
\hline & & REF & & & .03 & .34 \\
\hline \multirow{7}{*}{ BDI-II } & 1 & PSWQ & .20 & $42.84_{1164 * * *}$ & .45 & $6.54 * * *$ \\
\hline & 2 & PSWQ & .31 & $26.48_{(1,163)}^{(1,164)} * * *$ & .19 & $2.32 *$ \\
\hline & & ONP & & & .42 & $5.14 * * *$ \\
\hline & 3 & PSWQ & .42 & $16.48_{(2,161)} * * *$ & .013 & .16 \\
\hline & & ONP & & & .22 & $2.68 * *$ \\
\hline & & REP & & & .49 & $5.20^{* * *}$ \\
\hline & & REF & & & -.02 & -.335 \\
\hline \multirow{7}{*}{ BAI } & 1 & PSWQ & .32 & $79.89 \quad * * *$ & .56 & $8.93 * * *$ \\
\hline & 2 & PSWQ & .37 & $15.58_{(1,166)}^{(1,16)} * * *$ & .37 & $4.75 * * *$ \\
\hline & & ONP & & & .31 & $3.94 * * *$ \\
\hline & 3 & PSWQ & .37 & $.52_{(2,164)}$ & .34 & $3.89 * * *$ \\
\hline & & ONP & & & .27 & $3.17 * * *$ \\
\hline & & REP & & & .08 & .86 \\
\hline & & REF & & & .00 & .07 \\
\hline \multirow{7}{*}{ TEP } & 1 & PSWQ & .13 & $25.97_{(1,165)} * * *$ & .36 & $5.09^{* * *}$ \\
\hline & 2 & PSWQ & .17 & $9.57_{(1,166)}^{(1,165) * *}$ & .19 & $2.11 *$ \\
\hline & & ONP & & & .28 & $3.09 * * *$ \\
\hline & 3 & PSWQ & .27 & $11.46_{(2,164)} * * *$ & .00 & .07 \\
\hline & & ONP & & & .10 & 1.08 \\
\hline & & REP & & & .44 & $4.11^{* * *}$ \\
\hline & & REF & & & 01 & \\
\hline
\end{tabular}

Nota . $\mathrm{ONP}=$ Orientación negativa al problema; $\mathrm{REP}=$ Reproches, $\mathrm{REF}=$ Reflexión; $\mathrm{PSWQ}=$ Rasgo de preocupación; $\mathrm{FOB}=$ Fobia Social, DIE $=$ Dieta; TAG $=$ Trastorno de ansiedad generalizada; $\mathrm{CON}=$ Control; BDI-II $=$ depresión: BAI= Agorafobia y pánico; TEP $=$ Trastorno de estrés postraumático; $*=\mathrm{p} \leq .05 ; * *=\mathrm{p} \leq .01 ; * * *=\mathrm{p} \leq .001$. 
para la fobia social. Finalmente, para la bulimia ninguna de las variables consideradas parece ser relevante.

\section{DISCUSIÓN}

Los resultados de este trabajo aportan mayor consistencia a lo encontrado previamente sobre los procesos cognitivos de preocupación, reproches y orientación negativa al problema como constructos transdiagnósticos relacionados con los síntomas de ansiedad, depresión y dificultades de la conducta alimentaria (McEvoy et al., 2013; McLaughlin \& Nolen-Hoeksema, 2011; Nolen-Hoeksema et al., 2008).

Las relaciones entre reproches y reflexión se encuentran dentro de los rangos de estudios previos (D'Hudson, Lauren \& Saling, 2010), pero, aunque relacionados, se constató la independencia entre ellos con variables psicopatológicas. Este resultado sugiere que es importante analizar esos dos constructos de forma separada tanto en estudios concurrentes como longitudinales, esto dado, además, a que existen dos formas diferentes en su funcionalidad (Schoofs, Hermans $\&$ Reas, 2010; Treynor et al., 2003).

También se constató una relación de mayor peso entre los reproches y la depresión que entre los reproches y la sintomatología ansiosa, mientras que la reflexión muestra las relaciones más bajas con los variables antes mencionadas (D'Hudson et al., 2010; Hasegawa et al., 2015; McEvoy \& Brans, 2013) debido, posiblemente, a que esta última sea una estrategia más adaptativa (Treynor et al., 2003). Sin embargo, esta situación cambia a nivel predictivo, pues los reproches predicen tanto el estrés postraumático como la depresión, $\mathrm{y}$, de hecho, con un peso más elevado en la primera. Este resultado es coherente con los modelos teóricos del estrés postraumático, que se caracterizan por un estilo de procesamiento cognitivo emocional sobre el trauma y sus consecuencias, y en donde se reprocha cómo es que el suceso pudo haberse evitado y cómo se van a vengar o intentarán hacer justicia; pensamientos intrusivos que se relacionan con el trauma. Dichos reproches pueden ser la representación de una estrategia de evitación cognitiva que interfiriere con la memoria del trauma e impide el procesamiento emocional del mismo (Michael, Halligan, Clark \& Ehlers, 2007).

Con respecto a las dificultades de la conducta alimentaria, los reproches predijeron la dieta; resultado coherente con las conductas evitativas asociadas a los alimentos que engordan, dado que la no consecución de esas metas o estándares van acompañadas, específicamente, de los reproches. Sin embargo, la preocupación predijo el control, el cual viene motivado por la ausencia de autocontrol de la ingesta y la preocupación por el peso, la figura y la delgadez; resultado coherente con lo encontrado en investigaciones previas (Danzilo et al., 2016).

Por otra parte, la preocupación presenta relaciones más elevadas con los síntomas de ansiedad (González et al., 2006), excepto en el estrés postraumático y las dificultades de la conducta alimentaria. Particularmente, las regresiones realizadas indican que esta es la variable que mejor predice los síntomas de ansiedad (McLaughlin \& Nolen-Hoeksema, 2011). Esto puede deberse a que las preocupaciones se orientan más hacia los problemas que se están percibiendo como amenazantes, y no tanto hacia las lamentaciones sobre uno mismo o las autocríticas, como se podría esperar en la depresión (McLaughlin et al., 2007; Watkins et al., 2005).

También, la orientación negativa al problema se relacionó de manera similar con los síntomas de ansiedad y depresión (González et al., 2004; Hasegawa et al., 2015). Sin embargo, en el nivel predictivo tiene un peso más elevado en la fobia social, la agorafobia y pánico, el trastorno de ansiedad generalizada, y la depresión. Resultado que corrobora las fuertes relaciones encontradas entre preocupación y la orientación negativa al problema, donde las personas emplean las primeras como estrategia de afrontamiento a los problemas.

Por otra parte, en cuanto al género, los resultados no confirman lo encontrado sobre que las mujeres presentan mayores puntuaciones en depresión, orientación negativa al problema, reproches y reflexión (Lyubomirsky et al., 2014; Hasegawa et al., 2015), aunque sí se encuentra que estas obtienen puntuaciones medias más elevadas en preocupación y trastorno de ansiedad generalizada en comparación con los hombres (González, Rovella, Barbenza \& Rausch, 2012). Sin embargo, sí se encuentra consistencia con lo encontrado en otras investigaciones donde no se evidenciaron diferencias por sexo en rumiación, reproches y reflexión (Watkins, 2009). Este resultado podría explicarse tentativamente porque en el presente estudio, al trabajarse con una muestra de la población extrauniversitaria y de la comunidad, tanto los hombres como las mujeres presentaron un mismo nivel de estudios, ambos se encontraban trabajando y sus edades eran similares.

Específicamente, se encontró que la reflexión es un predictor de la fobia social, lo que podría explicarse teniendo en cuenta que los modelos teóricos de la ansiedad social han propuesto que las personas socialmente ansiosas participan en un proceso reflexivo posterior al evento social denominado procesamiento posterior al evento (Rapee \& Heimberg, 1997); en esta reflexión, la persona realiza un análisis muy detallado sobre cómo ha actuado en la situación social, y suele centrarse selectivamente en los elementos negativos. Este proceso se ha agrupado en la categoría de la rumiación (Wong \& Moulds, 2009), y es, por tanto, 
transdiagnóstico al trastorno de ansiedad generalizada, la agorafobia y pánico, y el trastorno obsesivo compulsivo (Laposa, Collimore \& Rector, 2014).

Adicionalmente, las definiciones de la rumiación y de la preocupación tienen en común un déficit en la resolución de problemas activos, $y$ las personas creen que si se preocupan o rumian sobre sus estados aversivos resuelven su malestar emocional, de manera que las personas se adhieren a la preocupación para prepararse frente a las amenazas futuras y evitar los estados emocionales negativos (Borkovec, Alcaine \& Behar, 2004).

Por otra parte, es posible que las personas conecten a la rumiación como una opción llamativa para escapar o evitar sus problemas, lo que a su vez conllevaría a presentar más afecto negativo, y este a más rumiación; de este modo, se estaría perpetuando el ciclo de la rumiación al no encontrar una solución activa al estado afectivo. Por estas razones, la rumiación puede considerarse como una estrategia de evitación cognitiva implicada en diversos trastornos psicopatológicos (Aldao, Nolen-Hoeksema \& Schweizer, 2011).

Esta perspectiva de la evitación cognitiva de la preocupación está tomando fuerza en las teorías actuales de la rumiación (Dickson et al., 2012). En este sentido, parece que tanto la rumiación como la preocupación actuarían como procesos de evitación cognitiva, por lo que la Teoría de los estilos de respuestas se podría beneficiar de la Teoría de la evitación de la preocupación, y, de este modo, podría prevenirse o tratarse el trastorno de ansiedad generalizada (Borkovec et al., 2004); por ello, los estudios futuros deben centrarse en si los reproches y la reflexión están relacionadas con determinadas estrategias de evitación cognitiva.

Para finalizar, a pesar de los aportes encontrados, este estudio presenta algunas limitaciones, tales como haber contado con una muestra pequeña e incidental; presentar una distribución de las puntuaciones con una asimetría positiva en las variables psicopatológicas que hace difícil generalizar los resultados; y el hecho de que se trate de un estudio transversal que no explica la causalidad de los procesos cognitivos en relación con los trastornos psicopatológicos evaluados, tal como ocurriría si se tratara de un estudio longitudinal.

\section{REFERENCIAS}

Aldao, A., Nolen-Hoeksema, S., \& Schweizer, S. (2011). Emotion-regulation strategies across psychopathology: A metaanalytic review. Clinical Psychology Review, 30(2), 217237. DOI: https://doi.org/10.1016/j.cpr.2009.11.004.

Arditte, K. A., Sahw, A. S., \& Timpano, K. (2016). Repetitive negative thinking: a transdiagnostic correlate of affective disorders. Journal of Social and Clinical Psychology, 35(2), 181-201. DOI: https://doi.org/10.1521/jscp.2016.35.3.181.

Beck, A. T., Epstein, N., Brown, G., \& Steer, R. A. (1988). An inventory for measuring clinical anxiety: Psychometric properties. Journal of Consulting and Clinical Psychology, 56(6), 893-897. DOI: https://doi.org/10.1037/0022006X.56.6.893.

Beck, A. T., Steer, R. A., \& Brown, G. K. (1996). Manual for the Beck Depression Inventory, Second Edition. San Antonio, TX: The Psychological Corporation. DOI: https://doi. org/10.1037/0022-006X.56.6.893.

Boelen, P. A., Reijntjes, A., \& Smid, G. E. (2016). Concurrent and prospective associations of intolerance of uncertainty with symptoms of prolonged grief, posttraumatic stress, and depression after bereavement. Journal of Anxiety Disorders, 41, 65-72. DOI: https://doi.org/10.1016/j.janxdis.2016.03.004.

Borkovec, R., Robinson, E., Pruzinsky, T., \& Depree, J. (1983). Preliminary exploration of worry: Some characteristics and processes. Behaviour Research and Therapy, 21(1), 9-16. DOI: https://doi.org/10.1016/0005-7967(83)90121-3.

Borkovec, T. D., Alcaine, O. M., \& Behar, E. (2004). Avoidance theory of worry and generalized anxiety disorder. En R. G., Heimberg, C. L., Turk, \& D. S., Mennin, (Eds.), Generalized Anxiety Disorder: Advances in research and practice (pp. 77-108). New York: Guilford Press.

Brown, T. A., White, K. S., \& Barlow, D. H. (2005). A psychometric reanalysis of the Albany Panic and Phobia Questionnaire. Behaviour Research and Therapy, 43(3), 337-355. DOI: https://doi.org/10.1016/j.brat.2004.03.004.

Danzilo, L., Rieger, E., Palermo, R., Byrne, S., \& Bell, J. (2016). Association between rumination factors and eating disorder behaviours in young women. Advances in Eating Disorders. Theory, Research and Practice, 4, 84-98. DOI: http://dx.doi.org/10.1080/21662630.2015.1118642

Dickson, K. S., Ciesla, J. A., \& Reilly, K. (2012). Rumination, Worry, Cognitive Avoidance, and Behavioral Avoidance: Examination of Temporal Effects. Behavior Therapy 43(3) 629-640. DOI: https://doi.org/10.1016/j.beth.2011.11.002.

Doninger, G. L., Enders, C. K., \& Burnett, K. F. (2005). Validity evidence for Eating Attitudes Test scores in a sample of female college athletes. Measurement in Physical Education and Exercise Science, 9(1), 35-49. DOI: https://doi. org/10.1207/s15327841mpee0901_3.

D’Hudson, G., Lauren, L., \& Saling, L. L. (2010). Worry and rumination in older adults: Differentiating the processes. Aging and Mental Health, 14(5), 524-534. DOI: https://doi. org/10.1080/13607861003713141.

Ehring T., \& Watkins, E. (2008). Repetitive negative thinking as a transdiagnostic process. International Journal of Cognitive Therapy, 1(3) 192-205. DOI: https://doi.org/10.1521/ ijct.2008.1.3.192.

Fergus, T. A., Valentine, D. P., Wu, K. D., \& McGrath, P. B. (2015). Examining the Symptom-Level Specificity of Neg- 
ative Problem Orientation in a Clinical Sample. Cognitive Behaviour Therapy, 44(2) 153-161. DOI: https://doi.org/10 $.1080 / 16506073.2014 .987314$.

Fisher, R. A. (1925). Statistical methods for research workers. Edinburgh, Scotland: Oliver and Boyd.

Garner, D. M., Olmsted, M. P., Bohr, Y., \& Garfinkel, P. E. (1982). The Eating Attitudes Test: psychometric features and clinical correlates. Psychological Medicine, 12(4), 871878. DOI: https://doi.org/10.1017/S0033291700049163.

González, M., Ibáñez, I., \& Cubas, R. (2006). Variables de proceso en la determinación de la ansiedad generalizada y su generalización a otras medidas de ansiedad y depresión. International Journal of Clinical and Health Psychology, 6(1), 23-39.

González, M., Peñate, W., Bethencourt, J. M., \& Rovella, A. (2004). La predicción del trastorno de ansiedad generalizada en función de variables de procesos. Psicología y Salud, 14(2), 179-188.

González, M., Rovella, A., Barbenza, C., \& Rausch, L. (2012). Tendencia a la Preocupación y Trastorno de Ansiedad Generalizada en adolescentes: contribución diferencial de los procesos cognitivos. Revista Iberoamericana de Diagnóstico y Evaluación Psicológica, 33(2), 31-50.

Hasegawa, A., Hattori, Y., Nishimura, H., \& Tanno, Y. (2015). Prospective associations of depressive rumination and social problem solving with depression: a 6-month longitudinal study. Psychological Reports, 116(3), 870-888. DOI: https://doi.org/10.2466/02.20.PR0.116k28w7.

Holm-Denoma, J. M., \& Hankin, B. L. (2011). Perceived physical appearance mediates the rumination and bulimic symptom link in adolescent girls. Journal of Clinical Child and Adolescent Psychology, 39(4), 537-544. DOI: https://doi.or $\mathrm{g} / 10.1080 / 15374416.2010 .486324$.

Johnson, D., \& Whisman, M. A. (2013). Gender differences in rumination: A meta-analysis. Personality and Individual Differences, 52(4), 367-374. DOI: https://doi.org/10.1016/j. paid.2013.03.019.

Jordan, J., Joyce, P. R., Carter, F. A., Horn, J., McIntosh, V. V. W., Luty, S. E., et al. (2008). Specific and nonspecific comorbidity in anorexia nervosa. International Journal of the Eating Disorders, 41(1), 47-56. DOI: https://doi. org/10.1002/eat.20463.

Kalmbach, D. A, Pillai, V., \& Ciesla, J. A. (2016). The correspondence of changes in depressive rumination and worry to weekly variations in affective symptoms: A test of the tripartite model of anxiety and depression in women. Australian Journal of Psychology, 68(1), 52-60. DOI: https:// doi.org/10.1111/ajpy.12090.

Kessler, R. (2006). Epidemiology of depression among women. En C. Keyes \& S. Goodman (Eds.), Women and depression: A handbook for the social, behavioral and biomedical science (pp. 22-37). New York, NY: Cambridge University Press. DOI: https://doi.org/10.1017/CBO9780511841262.004.
Laposa, J. M., Collimore, K. C., \& Rector, N. A. (2014). Is Post-Event Processing a Social Anxiety Specific or Transdiagnostic Cognitive Process in the Anxiety Spectrum? Behavioural and Cognitive Psychotherapy, 42(6), 706-717. DOI: https://doi.org/10.1017/S135246581300074X.

Londoño, C., Peñate, W., \& González, M. (2016). Validación Diferencial y Discriminante del Cuestionario de Depresión para Hombres (CDH). Acta Colombiana de Psicología, 34(2), 169-179. DOI: http://www.dx.doi.org/10.14718/ ACP.2016.19.2.13

Lyubomirsky, S., Chancellor, J., Layous, K., \& Nelson. K. S. (2014). Thinking About Rumination: The Scholarly Contributions and Intellectual Legacy of Susan Nolen-Hoeksema. Annual Review of Clinical Psychology, 11, 1-22. DOI: https://doi.org/10.1146/annurev-clinpsy-032814-112733.

McEvoy, P. M., \& Brans, S. (2013). Common Versus Unique Variance Across Measures of Worry and Rumination: Predictive Utility and Mediational Models for Anxiety and Depression. Cognitive Therapy and Research, 37(1), 183-196. DOI: https://doi.org/10.1007/s10608-012-9448-5.

McEvoy, P. M., Watson, H., Watkins, E. R., \& Nathan, P. (2013). The relationship between worry, rumination, and comorbidity: evidence for repetitive negative thinking as a transdiagnostic construct. Journal of Affective Disorders, 151(1), 313-320. DOI: https://doi.org/10.1016/j.jad.2013.06.014.

Meyer, T., Miller, M., Metzger, R., \& Borkovec, T. (1990). Development and validation of the Penn State Worry Questionnaire. Behaviour Research and Therapy, 28(6), 487495. DOI: https://doi.org/10.1016/0005-7967(90)90135-6.

McLaughlin, K. A., Borkovec, T. D., \& Sibrava, N. J. (2007). The effects of worry and rumination on affect states and cognitive activity. Behavior Therapy, 38(1), 23-38. DOI: https://doi.org/10.1016/j.beth.2006.03.003

McLaughlin, A., \& Nolen-Hoeksema, S. (2011). Rumination as a Transdiagnostic Factor in Depression and Anxiety. Behaviour Research and Therapy, 49(3), 186-193. DOI: https:// doi.org/10.1016/j.brat.2010.12.006.

Michael, T., Halligan, S. L., Clark, D., \& Ehlers, A. (2007). Rumination in Posttraumatic Stress Disorder. Depression and Anxiety, 24(5), 307-317. DOI: https://doi.org/10.1002/ da. 20228 .

Nolen-Hoeksema, S., \& Morrow, J. (1991). A prospective study of depression and posttraumatic stress symptoms after a natural disaster: the 1989 Loma Prieta earthquake. Journal of Personality and Social Psychology 61(1), 115-121. DOI: https://doi.org/10.1037/0022-3514.61.1.115.

Nolen-Hoeksema, S., \& Morrow, J. (1993). The effects of rumination and distraction on naturally-occurring depressed moods. Cognition and Emotion, 7(1), 561-570. DOI: http:// dx.doi.org/10.1080/02699939308409206.

Nolen-Hoeksema, S., Wisco, B. E., \& Lyubomirsky, S. (2008). Rethinking rumination. Perspectives on Psychological Science, 3(5), 400-424. DOI: https://doi.org/10.1111/ j.1745-6924.2008.00088.x. 
Pérez, G. B., García, F. L., De Vicente, M. P., \& Oliveras, V. A. (2010). Validación del Psychiatric Diagnostic Screening Questionnaire (PDSQ) en una muestra de pacientes alcohólicos españoles. Adicciones, 22(3), 199-206.

Rapee, R. M., \& Heimberg, R. G. (1997). A cognitive-behavioral model of anxiety in social phobia. Behaviour Research and Therapy, 38(8), 741-756. DOI: https://doi. org/10.20882/adicciones.180.

Rawal, A., Park, R. J., \& Williams, J. M. G. (2010). Rumination, experiential avoidance, and dysfunctional thinking in eating disorders. Behaviour Research and Therapy, 48(9) 851-859. DOI: https://doi.org/10.1016/j.brat.2010.05.009.

Robichaud, M., \& Dugas, M. J. (2005). Negative problem orientation (Part II): Construct validity and specificity to worry. Behaviour Research and Therapy, 43(3), 403-412. DOI: https://doi.org/10.1016/j.brat.2004.02.008.

Rodebaugh, T. L., Woods, C. M., \& Heimberg, R. G. (2007). The reverse of social anxiety is not always the opposite: the reverse-scored items of the social interaction anxiety scale do not belong. Behavior Therapy, 38(2), 192-206. DOI: https://doi.org/10.1016/j.beth.2006.08.001.

Schoofs, H., Hermans, D., \& Raes, F. (2010). Brooding and reflection as subtypes of rumination: evidence from confirmatory factor analysis in nonclinical samples using the Dutch Ruminative Response Scale. Journal of Psychopathology and Behavioral Assessment, 32(4), 609-617. DOI: https:// doi.org/10.1007/s10862-010-9182-9.

Segerstrom, S. C., Tsao, J. C. I., Alden, L. E. \& Craske, M. G. (2000). Worry and rumination: Repetitive thought as a concomitant and predictor of negative mood. Cognitive Therapy and Research, 24(6), 671-688. DOI: https://doi. org/10.1023/A:1005587311498.

Serra, J. A., \& Irizarry-Robles, C. Y. (2015). Factores protectores de la depresión en una muestra de adultos mayores en Puerto Rico: autoeficacia, escolaridad y otras variables socio-demográficas. Acta Colombina de Psicología, 18(1), 125-134. DOI: https://doi.org/10.14718/ACP.2015.18.1.12.

Spinhoven, P., Drost, J., Van Hemert, B., \& Penninx, B. W. (2015). Common rather than unique aspects of repetitive negative thinking are related to depressive and anxiety disorders and symptoms. Journal of Anxiety Disorders, 33, 4552. DOI: http://dx.doi.org/10.1016/j.janxdis.2015.05.001
Spitzer, R. L., Kroenke, K., Williams, J. B. W. \& Löwe, B. (2006). A Brief Measure for Assessing Generalized Anxiety Disorder: The GAD-7. Archives of Internal Medicine, 166(10) 1092-1097. DOI: https://doi.org/10.1001/ archinte.166.10.1092.

Sternheim, L., Startup, H., Saeidi, S., Morgan, J., Hugo, P., Russell, A., et al. (2012). Understanding catastrophic worry in eating disorders: Process and content characteristics. Journal of Behaviour Therapy and Experimental Psychiatry, 43(4), 1095-1103. DOI: https://doi.org/10.1016/j. jbtep.2012.05.006.

Thanoi, W., \& Klainin-Yobas, P. (2015). Assessing rumination response style among undergraduate nursing students: A construct validation study. Nurse Education Today, 35(5), 641-646. DOI: https://doi.org/10.1016/j.nedt.2015.01.001.

Thomson, S. K. (2002). Sampling. New York: John Wiley \& Sons, Inc.

Treynor, W., González, R., \& Nolen-Hoeksema, S. (2003). Rumiation reconsidered: A psychometric analysis. Cognitive Therapy and Research, 27(3), 247-259. DOI: https://doi. org/10.1023/A:1023910315561.

Watkins, E. (2009). Depressive rumination and co-morbidity: Evidence for brooding as a transdiagnostic process. Journl of Rational Emotive and Cognitive Behavioral Therapy, 27(3), 160-175. DOI: https://doi.org/10.1007/s10942-0090098-9.

Watkins, E., Moulds, M. \& Mackintosh, B. (2005). Comparisons between rumination and worry in a non-clinical population. Behaviour Research and Therapy, 43(12), 15771585. DOI: https://doi.org/10.1016/j.brat.2004.11.008.

Wong, Q. J. J., \& Moulds, M. L. (2009). Impact of rumination versus distraction on anxiety and maladaptive self-beliefs in socially anxious individuals. Behaviour Research and Therapy, 47(10), 861-867. DOI: https://doi.org/10.1016/j. brat.2009.06.014.

Zimmerman, M., \& Mattia, J. I. (2001). A Self-Report Scale to Help Make Psychiatric Diagnoses: The Psychiatric Diagnostic Screening Questionnaire. Archives of General Psychiatry,58(8), 787-94. DOI: https://doi.org/10.1001/ archpsyc.58.8.787. 